\title{
SOME REMARKS ON LINEAR DIFFERENTIAL SYSTEMS*
}

\author{
WILLIAM T. REID
}

1. Introduction. In 1926 Bliss $\dagger$ formulated a definition of selfadjoint and definitely self-adjoint systems of ordinary linear differential equations of the first order with two-point boundary conditions, the coefficients of the system being real. More recently, Bliss $\ddagger$ has modified the definition of definite self-adjointness in such a manner that the boundary value problems arising in a well known way from nonsingular problems of Bolza in the calculus of variations are definitely self-adjoint by the new definition. Further intimate relationships that exist between such definitely self-adjoint boundary value problems and the boundary value problems associated with the calculus of variations have been established by Reid.§

In this note linear differential systems with complex-valued coefficients are considered and self-conjugate adjoint and definitely selfconjugate adjoint systems are defined. It is shown that definitely self-conjugate adjoint systems have only real characteristic values, and that for a consideration of the questions of the existence of characteristic values and associated expansion theorems such a system may be replaced by a corresponding definitely self-adjoint system with real coefficients. In $\S 4$ there is solved an associated matrix differential equation, and the question of self-conjugate adjointness is reduced to the determination of a nonsingular constant matrix of a certain type. Finally, there is given an application of this matrix differential equation to the theory of matrix differential equations of Riccati type.

Matrix notation is used throughout. Capital italic letters denote $n$-rowed square matrices whose elements are complex-valued, the element in the $i$ th row and $j$ th column being denoted by the same letter with the subscript $i j$. Lower case italic letters signify vectors with $n$ components, the $i$ th component being denoted by a subscript $i$. If $M \equiv\left\|M_{i j}\right\|, u \equiv\left[u_{i}\right]$, the vectors $\left[M_{i j} u_{j}\right]$ and $\left[u_{j} M_{j i}\right]$ are denoted by $M u$ and $u M$, respectively, where the repetition of the subscript $j$ indi-

* Presented to the Society, September 6, 1938.

$\dagger$ Transactions of this Society, vol. 28 (1926), pp. 561-584. This paper will be referred to as B.I.

$\ddagger$ Transactions of this Society, vol. 44 (1938), pp. 413-428. This paper will be referred to as B.II.

$\S$ Transactions of this Society, vol. 44 (1938), pp. 508-520. 
cates summation with respect to this subscript over the range $1, \cdots, n$. The scalar product $u_{j} v_{j}$ of two vectors is denoted simply by $u v$. If $\alpha$ is a scalar, $\bar{\alpha}$ is its complex conjugate, and for a vector $u$ we write $\bar{u}$ for $\left[\bar{u}_{i}\right]$. For a matrix $M \equiv\left\|M_{i j}\right\|$ we use $\bar{M}$ for the conjugate matrix $\left\|\bar{M}_{i j}\right\|, \tilde{M}$ for the transpose matrix $\left\|M_{i i}\right\|$, and $M^{*}$ for the conjugate transpose $\left\|\bar{M}_{j i}\right\|$. Finally, if the elements of $M$ are differentiable functions, the matrix of derivatives is denoted by $M^{\prime}$; similarly, if the components of $u$ are differentiable functions, we write $u^{\prime} \equiv\left[u_{i}^{\prime}\right]$.

2. Self-conjugate adjoint systems. Using the notation of the preceding paragraph, the differential system which we shall consider involves the vector differential equations and the two-point boundary conditions

$$
y^{\prime}=[A(x)+\lambda B(x)] y, \quad s[y] \equiv M y(a)+N y(b)=0 .
$$

It will be supposed that the elements of $A$ and $B$ are continuous functions of the real variable $x$ on the finite interval $a b: a \leqq x \leqq b$, and that the constant matrices $M$ and $N$ are such that the relations $s_{i}[y]$ are linearly independent. If $\lambda$ is such that (2.1) has a solution $y(x) \not \equiv 0$ on $a b, \lambda$ is called a characteristic value, and such a solution is termed a characteristic solution of (2.1). If $p \equiv\left[p_{i}\right] \equiv\left[P_{i j}\right], q \equiv\left[q_{i}\right] \equiv\left[Q_{i j}\right]$, $(j=1, \cdots, n)$, form $n$ linearly independent solutions of the linear equations $M p-N q=0$, the system adjoint to (2.1) is (see B.I, p. 565),

$$
z^{\prime}=-z[A(x)+\lambda B(x)], \quad t[z] \equiv z(a) P+z(b) Q=0 .
$$

The system

$$
u^{\prime}=-u[\bar{A}(x)+\lambda \bar{B}(x)], \quad \bar{t}[u] \equiv u(a) \bar{P}+u(b) \bar{Q}=0
$$

we shall term the conjugate of (2.2), and the conjugate adjoint of (2.1). Clearly if the elements of $A(x), B(x), M$, and $N$ are real, the elements of $P$ and $Q$ may be chosen real, and in this case system (2.3) is identical with (2.2). In general, if $u=u(x)$ is a solution of (2.3) for a value $\lambda$, then $z=\bar{u}(x)$ is a solution of (2.2) for the corresponding conjugate value $\bar{\lambda}$.

The system (2.1) will be called self-conjugate adjoint if it is equivalent to (2.3) under a linear transformation $u=T(x) y$, where the elements of $T$ are complex-valued functions which are continuous and have continuous derivatives on $a b$, and the determinant of $T$ is different from zero on this interval. For brevity, a nonsingular matrix $T(x)$ whose elements have continuous derivatives on $a b$ will be termed an admissible transformation matrix. 
THEOREM 2.1. The system (2.1) is self-conjugate adjoint if and only if there is an admissible transformation matrix such that

$$
\begin{gathered}
T A+A^{*} T+T^{\prime} \equiv 0, \quad T B+B^{*} T \equiv 0, \\
M T^{-1}(a) M^{*}=N T^{-1}(b) N^{*} .
\end{gathered}
$$

Relations (2.4) follow immediately upon the substitution of $u=T y$ in (2.3). In order for the linear equations $s[y]=0$ to be equivalent to the set $\bar{t}[u]=0$ under this transformation, it is seen to be necessary and sufficient that there exist a nonsingular constant matrix $C$ such that

$$
C P^{*} T(a)=M, \quad C Q^{*} T(b)=N,
$$

and since $P^{*} M^{*}-Q^{*} N^{*}=0$, it is readily seen that (2.5) is equivalent to (2.6). If the coefficients of the system (2.1) are real and $T$ is assumed to be real, conditions (2.4), (2.5) are of course exactly those obtained by Bliss (B.I, p. 569).

3. Definitely self-conjugate adjoint systems. Corresponding to the modified definition of definitely self-adjoint systems with real coefficients as given by Bliss in B.II, we say that the system (2.1) is definitely self-conjugate adjoint if there is an admissible transformation matrix $T$ with respect to which it is self-conjugate adjoint, and the corresponding matrix $S(x) \equiv T^{*}(x) B(x)$ satisfies the conditions:

(i) $S(x)$ is positive semi-definite and hermitian; that is, $S(x) \equiv S^{*}(x)$ and for arbitrary vectors $y$ the form $\bar{y} S(x) y$ is nonnegative.

(ii) If $y(x) \not \equiv 0$ is a solution of system (2.1) for some value $\lambda$, then $\bar{y}(x) S(x) y(x) \not \equiv 0$ on $a b$.

THEOREM 3.1. All the characteristic values of a definitely self-conjugate adjoint system (2.1) are real.

For if $y(x)$ is a characteristic solution of (2.1) corresponding to a characteristic value $\lambda$, then $z \equiv \bar{T}(x) \bar{y}(x)$ is a solution of (2.2) for the conjugate value $\bar{\lambda}$. From the differential equations of (2.1) and (2.2) we obtain

$$
\left.z(x) y(x)\right|_{a} ^{b}=(\lambda-\bar{\lambda}) \int_{a}^{b} z(x) B(x) y(x) d x .
$$

Since $s[y]=0$ and $t[z]=0$, we also have $\left.z(x) y(x)\right|_{a} ^{b}=0 . \dagger$ If $\lambda$ is not real, we then have

† See B.I, p. 565. Obviously the derivation of the formula (7) of B.I does not involve the reality of the elements of $M$ and $N$. 


$$
\begin{aligned}
0=\int_{a}^{b} z(x) B(x) y(x) d x & =\int_{a}^{b} \bar{y}(x) T^{*}(x) B(x) y(x) d x \\
& =\int_{a}^{b} \bar{y}(x) S(x) y(x) d x
\end{aligned}
$$

which, in view of the above hypotheses (i) and (ii), is impossible. Hence all the characteristic values of (2.1) are real.

Without loss of generality one may replace in (2.1) the parameter $\lambda$ by $\lambda \alpha$ where $\alpha$ is an arbitrary nonzero complex number. This is equivalent to replacing the matrix $B(x) \equiv\left\|B_{i j}(x)\right\|$ by $\alpha B(x) \equiv\left\|\alpha B_{i j}(x)\right\|$. We therefore have the following theorem:

THEOREM 3.2. Suppose that there exists a nonzero complex number $\alpha$ and an admissible transformation matrix $T(x)$ satisfying

$$
T A+A^{*} T+T^{\prime} \equiv 0, \quad \alpha T B+\bar{\alpha} B^{*} T \equiv 0,
$$

the condition (2.5), and that the matrix $S_{\alpha}(x)=\alpha T^{*} B$ satisfies conditions (i) and (ii) of $\$ 3$. Then all the nonzero characteristic values $\lambda$ of (2.1) have $\arg \lambda=\arg \alpha$.

As for the case of a definitely self-adjoint system with real coefficients (see B.II), one may proceed directly to show that for a definitely self-conjugate adjoint system the index of a characteristic value is equal to its multiplicity, and derive certain expansion theorems in terms of its characteristic solutions. In view of Theorem 3.1, however, the consideration of the existence of characteristic values and related expansion theorems for a definitely self-conjugate adjoint system (2.1) is reducible to the same consideration for an associated definitely self-adjoint system with real coefficients.

Corresponding to a square $n$-rowed matrix $K \equiv\left\|K_{i j}\right\| \equiv$ $K_{i j}^{1}+(-1)^{1 / 2} K_{i j}^{2} \|$ the corresponding script letter $K$ shall denote the square $2 n$-rowed matrix

$$
K \equiv\left\|\begin{array}{cc}
K^{1} & -K^{2} \\
K^{2} & K^{1}
\end{array}\right\|_{1}
$$

of real elements. Corresponding to a vector $y \equiv\left[y_{i}\right] \equiv\left[y_{i}{ }^{1}+(-1)^{1 / 2} y_{i}{ }^{2}\right]$, $\eta$ shall be defined by $\eta \equiv\left[y_{1}^{1}, \cdots, y_{n}^{1}, y_{1}^{2}, \cdots, y_{n}^{2}\right]$. Separating real and pure imaginary parts of (2.1) for real values of $\lambda$, we may write this system as

$$
\eta^{\prime}=[\mathcal{A}+\lambda \mathcal{B}]_{\eta}, \quad \mathscr{X}_{\eta}(a)+\mathcal{N} \eta(b)=0 .
$$

If the system (2.1) is self-conjugate adjoint with respect to an ad. 
missible transformation matrix $T(x)$, then the corresponding matrix $\mathcal{T}(x)$ is an admissible transformation matrix with respect to which (3.2) is self-adjoint. Moreover, if (2.1) is definitely self-conjugate adjoint with respect to an admissible transformation matrix $T(x)$, then the real system (3.2) is definitely self-adjoint with respect to $\mathcal{T}(x)$. The relations (2.4), (2.5) and conditions (i), (ii) may be verified directly. They are, however, immediate consequences of the fact that the algebra of square $n$-rowed matrices $K$ with complex elements is equivalent to the algebra of square $2 n$-rowed matrices $K$ of the form (3.1), with real elements.

4. A special matrix differential equation. In $\$ 2$ we have shown that conditions (2.4) and (2.5) are necessary and sufficient for system (2.1) to be self-conjugate adjoint with respect to an admissible transformation matrix $T(x)$. The first relation of (2.4) is a matrix differential equation, and, in particular, $T(x)$ must be a solution of this equation with determinant different from zero on $a b$. This matrix differential equation may obviously be considered as a linear vector differential system in the $n^{2}$ elements $T_{i j}(x),(i, j=1, \cdots, n)$. The condition that $\left|T_{i j}(x)\right| \neq 0$ on $a b$ is, however, a property of a single solution of this equation. The result that we wish is contained as a special instance of the following theorem:

TheOREM 4.1. Suppose the elements of the matrices $H(x), K(x)$ are continuous functions of $x$ on ab. Then the general solution $T(x)$ of the matrix differential equation

$$
T^{\prime}=H(x) T+T K(x)
$$

is of the form $T(x)=U(x) C V(x)$, where $U(x)$ is a fundamental matrix solution $\dagger$ of $U^{\prime}=H(x) U, V(x)$ is a fundamental matrix solution of $V^{\prime}=V K(x)$, and $C$ is an arbitrary constant matrix. In particular, if $\left|T\left(x_{0}\right)\right|=0$ for $a$ point $x_{0}$ of $a b$, then $|T(x)| \equiv 0$ on $a b$.

To prove the general form of $T(x)$ we have merely to write $T(x)=U(x) W(x)$, where $U(x)$ is a fundamental matrix solution of $U^{\prime}=H(x) U$. Upon substituting in (4.1) we obtain $U W^{\prime}=U W K$, which, since $|U| \neq 0$ on $a b$, is equivalent to $W^{\prime}=W K$. But the general solution of this last equation is $W=C V$, where $C$ is a constant matrix and $V$ is a fundamental matrix solution of $V^{\prime}=V K(x)$. Since

$$
|T(x)|=|U(x)| \cdot|C| \cdot|V(x)|
$$

and $|U(x)| \neq 0,|V(x)| \neq 0$ on $a b$, the rest of the theorem is immediate.

$\dagger$ That is, a solution with $|U(x)| \neq 0$ on $a b$. 
Applying this result to the matrix differential equation of (2.4), we have $H=-A^{*}, K=-A$. Moreover, it is seen that if $V(x)$ is a fundamental matrix solution of $V^{\prime}=-V A(x)$, then $U=V^{*}(x)$ is a fundamental matrix solution of $U^{\prime}=-A^{*}(x) U$. Hence the general form of the solution of this equation is $T(x)=V^{*}(x) C V(x)$. Consequently, the question of whether or not there is an admissible transformation matrix $T$ with respect to which system (2.1) is self-conjugate adjoint is equivalent to the question of whether or not there is a nonsingular constant matrix $C$ which satisfies with a fundamental matrix solution $V(x)$ of $V^{\prime}=-V A(x)$ the conditions

$$
\begin{gathered}
V^{*}(x) C V(x) B(x)+B^{*}(x) V^{*}(x) C^{\prime} V(x) \equiv 0, \\
M V^{-1}(a) C^{-1} V^{*-1}(a) M^{*}=N V^{-1}(b) C^{-1} V^{*-1}(b) N^{*} .
\end{gathered}
$$

In conclusion, we shall give an application of Theorem 4.1 to nonlinear matrix differential equations of the form

$$
Y^{\prime}+Y E(x) Y=R(x),
$$

where the elements of $E(x)$ and $R(x)$ are continuous on the interval $a b$.

TheOREM 4.2. If $Y=Y_{1}(x), Y=Y_{2}(x)$ are solutions of (4.3) whose elements are continuous on the interval $a b$, and if $T(x) \equiv Y_{1}(x)-Y_{2}(x)$ is such that $\left|T\left(x_{0}\right)\right|=0$ at a point $x_{0}$ of $a b$, then $|T(x)| \equiv 0$ on $a b$.

It may be verified directly that $T \equiv Y_{1}-Y_{2}$ satisfies (4.1) with

$$
\begin{aligned}
& H(x)=-(1 / 2)\left[Y_{1}(x)+Y_{2}(x)\right] E(x), \\
& K(x)=-(1 / 2) E(x)\left[Y_{1}(x)+Y_{2}(x)\right] ;
\end{aligned}
$$

hence Theorem 4.2 is an immediate consequence of Theorem 4.1 .

The hypotheses of a theorem due to W. M. Whyburn $†$ can be substantially weakened if use is made of the above theorem. Whyburn considered the matrix equation of Riccati type

$$
Y^{\prime}+Y Y=R(x)
$$

and stated specifically $\ddagger$ the assumption that the determinant of the difference of two solutions of this equation be different from zero on the entire interval considered. In view of the above theorem one may simply assume that this determinant is not identically zero on the interval.

\section{The University of Chicago}

† American Journal of Mathematics, vol. 56 (1934), pp. 587-592.

$\ddagger$ See loc. cit., Theorem 4, p. 590 . 